\title{
Efficient Cluster Elimination Protocol (ECEP) for Ad Hoc Networks
}

\author{
Tanu Preet Singh \\ Department of Computer Science \\ \& Engineering, Amritsar College \\ of Engineering \& Technology, \\ Punjab Technical University, \\ Jalandhar, India.
}

\author{
R.K. Singh \\ PhD, Department of \\ Electronics \& Communication \\ University, Uttrakhand \\ Technical University, \\ Dehradun, India.
}

\author{
Vishal Sharma \\ Department of Computer Science \& \\ Engineering, Amritsar College of \\ Engineering \& Technology, \\ Punjab Technical University, \\ Jalandhar, India.
}

\begin{abstract}
MANETs are networks capable of communicating in a set of small, low cost, low power sensing devices. A wireless sensor networks is totally based on the limiting factor i.e. energy consumption. A wireless sensor network consists of large number of sensor nodes distributed or scattered in particular network region. MANETs consist of node that is highly mobile, so in particular the range of the nodes is very important. Each device in a MANETs is free to move independently in any direction, and will therefore change its links to other devices frequently. The energy and the bandwidth of such path are of major concern. The lifetime of the network depends upon these parameters. There has been a paper already published by us termed DSPO that resolved the performance issues regarding the bandwidth efficiency and the energy efficiency. Also it suggested the alternatives to remove the dead state of the mobile nodes. But one of the concerns that arise as a future work of that protocol was the handling of delays that may arise and make the network incompetent in terms of the real time performance. In this paper, we are introducing the protocol that defines certain techniques that can be used to resolve the delays that may occur on the selection of alternative path during dynamic routing.
\end{abstract}

\section{Keywords}

Cluster, delays, bandwidth, throughput, network efficiency.

\section{INTRODUCTION}

A wireless ad hoc network is based on the nodes that are mobile and have capabilities of communicating each other with packet radios over a shared wireless medium. The limited radio propagation causes the route to be multi hop [1] [2] [7] [8]. The applications of such networks can be search and rescue, automated battlefields, disaster recovery, crowd control, and sensor networks. The routing protocol must have the ability to manage the frequent topology changes caused by the mobility of nodes and these need to be efficient as compared on basis of efficiency in terms of bandwidth and power as well as on basis of load transmission [13] [11]. With the advent of On-demand routing, the tables are not maintained and the topological views are also rescued and the routing totally becomes dynamic [11] [24]. Existing on demand routing protocols such as DSR (Dynamic Source Routing), AODV (Ad-hoc on demand distance vector routing) are the shortest path based routing protocols, also these don't consider the packet size and the antenna range of the nodes as a performance metric due to which there is a problem of long delays and congestions in the routing path and the whole set up of the nodal structure enters in to the dead state [10]. Also, on demand protocols that use the shortest paths as performance metric suffer from performance degradation as the network traffic increases [10]. In the paper [6], the energy of the nodes is the major area of concerned for the research to be carried on in this field. The one of the method suggested was CPACL protocol. It stands for cost based power aware routing protocol. In this paper the energy factor of the nodes is taken to be major concern. In this paper, a routing algorithm has been suggested that selects the path form the source to the destination on basis of the path that consumes the least energy [10]. The path selected for this transmission is the best selected path for the particular types of nodes. This means that if a path is defined from node 1 to node 2 by CPACL algorithm, it is the best suited path in all conditions [7] [10]. This protocol is the reactive routing protocol. It maintains the established routes as long as they are needed by the sources. AODV- CPACL uses sequence numbers to ensure the freshness of routes. The route discovery process is initiated whenever; a traffic source needs a route to a destination. Route discovery typically involves a network wide flood of route request packets targeting the destination and waiting for a route reply. It has also been shown that the per node throughput capacity of ad hoc networks with nodes $n$ decreases with $n$ as $\Theta(1 / n \log n){ }^{1 / 2}$ [3]. The issue regarding this has been shown as the general capacity cost function of channel capacity for arbitrary input alphabets was studied on single link [4]. In the related work the bits per joule capacity of the network is assumed [3]. The tradeoff between energy and the bandwidth has been analyzed under various assumptions on the channel condition and the interference under a linear equidistant relaying network model without considering the energy consumption at the receiver end [5][6][7]. Also the receiver consumption can be improved by using the cross layer design including the effects of the power amplifier used at the transmitter end [8]. The transport efficiency of an ad hoc network was defined considering the transmitter energy and the receiver's processing energy [9] [10]. Thus the energy consumption for the packet transmission and the large number of hops is considered [6]. For the networks that have energy as their limiting resource, the network lifetime related to the energy is one of the significant performance metrics [6]. To solve the dead state problem, we have earlier got our paper published that resolved this issue of nodes getting into the dead state. This protocol was termed as ARPA. But there was a concern about delays that may arise due to selection of alternative path that may occur as 
hindrance for the real time networks that have performance issues with them.

In this paper we are introducing a new protocol that suggests certain techniques that can be applied to resolve the issues regarding the delays. The work carried out by has been simulated using the NS-2 simulator. The paper also includes the working parameters and the concept of configuring the NS-2 for obtaining the desired scenario and the traffic generation to show the correctness of protocol.

\section{SYSEM MODEL}

The network model we considered comprises of $\mathrm{k}$ number of hops, hops here are the nodes, and the nodes here considered are to be single channel node. This means for $\mathrm{k}$ number of nodes there is $\mathrm{k}$ number of channels. Thus, if two nodes are communicating at a time, then we have k-1 number of relaying nodes in the network model. The distance between the source and the destination is denoted by $\mathrm{d}$. the distance between the relaying nodes can be decided on basis of the dynamic routing considered or it can be given on mathematical computations, this means that the distance between the relaying nodes will be less than the actual distance between the source and destination. Thus, if we consider a constant, let this constant be $\alpha$ n then, from the theoretical analysis [6], we obtain that this value is multiplied with the total distance to obtain the actual distance between the relaying nodes then this value should be positive and less than one. Thus, the distance between the relaying nodes will be:

$$
\mathrm{de}=\sum_{n=0}^{k} \alpha \mathrm{d}
$$

The mobility introduces another simple concept. If the mobility of the structure nodes is more, the attenuation has greater effect but if the nodes are considered to be at rest then, the attenuation comes out to be so small that it can be neglected. Thus, the modified formula for the attenuation loss in a network model will be:

$$
\operatorname{Pr}=\beta \frac{\text { Pout }}{d \eta} \zeta \S
$$

Here, is the attenuation loss in the MANETSs, $\beta$ is the antenna constant, $\mathrm{d}$ is the end to end distance between source and destination, $\varepsilon$ is the path loss constant such that $2<\varepsilon<4$ and $\delta$ is the mobility factor. The mobility can be computed by analyzing the movement in terms of number of bits transferred per second per meter of the network model. Here, Pout $=$ fo (Pin), which is based on the working power amplifier present in each of the node.

\section{CLUSTER ELIMINATON}

The most important reason for the mobile nodes to contain large amount of delays is the reason of formation of clusters. Thus, delays can be controlled if nodes are not allowed to come together to form highly cluster zone that can reduce the link speed and thus decrease the network performance by increasing the delays. The cluster formation can be reduce by use of special signals to be transmitted from nodes that will send its latitudinal and longitudinal location in those signals thus, any node that will be nearby can catch those signals and interpret the position of node an thus will not form clusters. This technique will maintain a certain distance limit between the nodes and the link speed will also be increased that will reduce the delays that are liable to occur in the network model.
The delays are bound to occur when the traffic as well as the number of nodes is more in a limited geographical area. Thus, taking this thing into account, we developed a protocol that incorporates this problem and provides a zero delay solution in case of formation of cluster during the transmission process. The technique was shown for lesser number of nodes to clearly state what actually happens in case of delay formation. Firstly, we will be dealing with the actual formation of delays and then coming up with the solution to eradicate such problem.

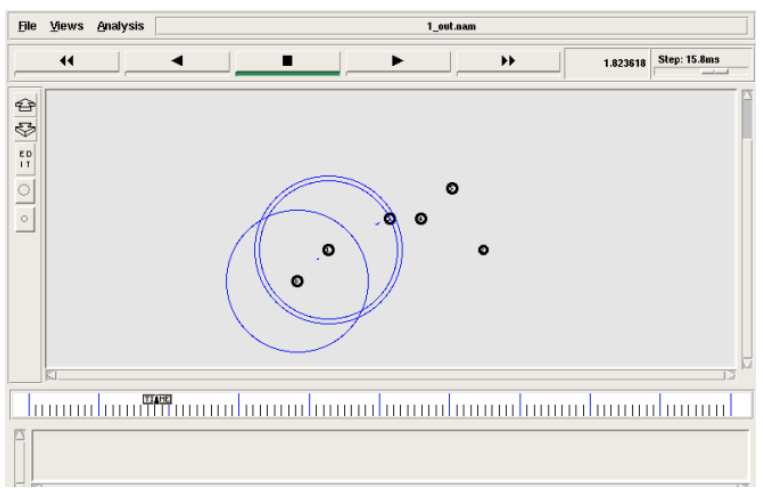

Fig. 6

The Fig. 6 shows the initial state comprising of six nodes out of which the three are communicating with each other on basis of AODV as it base protocol.

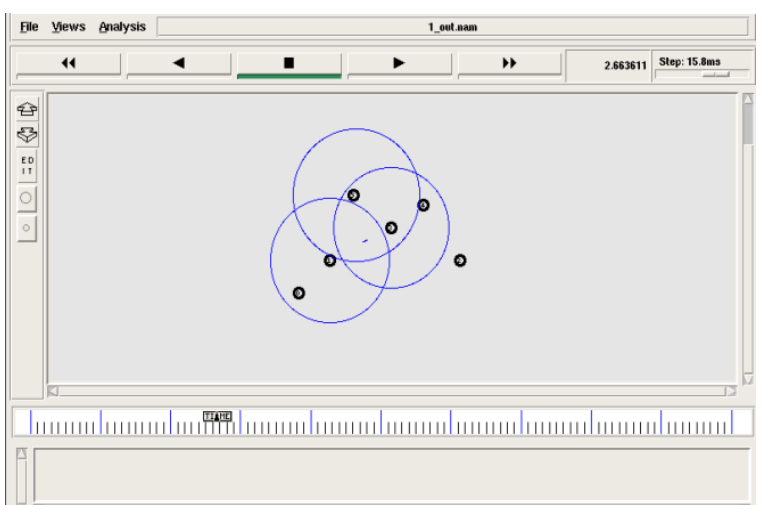

Fig. 7

Clearly, notice that the nodes have shown mobility as the original location of nodes has changed that can be evaluated by comparing with the Fig.6. now the transmission path has also changed as it is a property of AODV protocol.

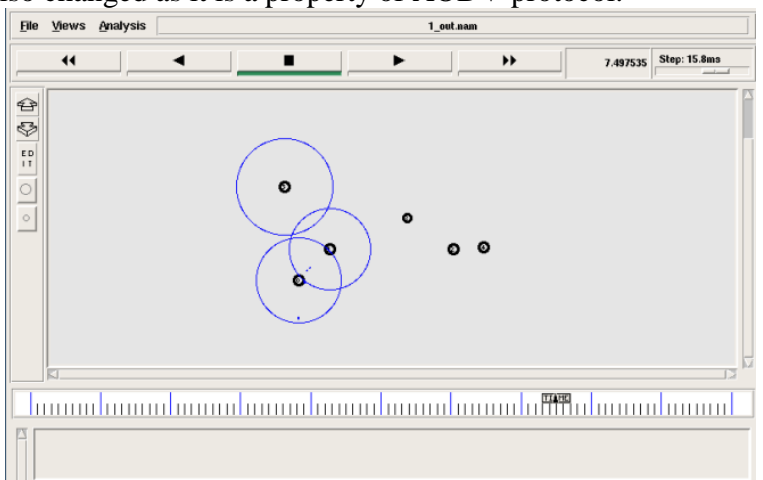

Fig. 8 
Fig. 8 shows that two of the six nodes have gone to extreme positions as the kept on changing locations in the decided geographical area. But what if any of the above nodes interfere with the relaying node that forms a path between the receiver and the transmitter, this may lead to halt in transmissions as shown in Fig. 9.

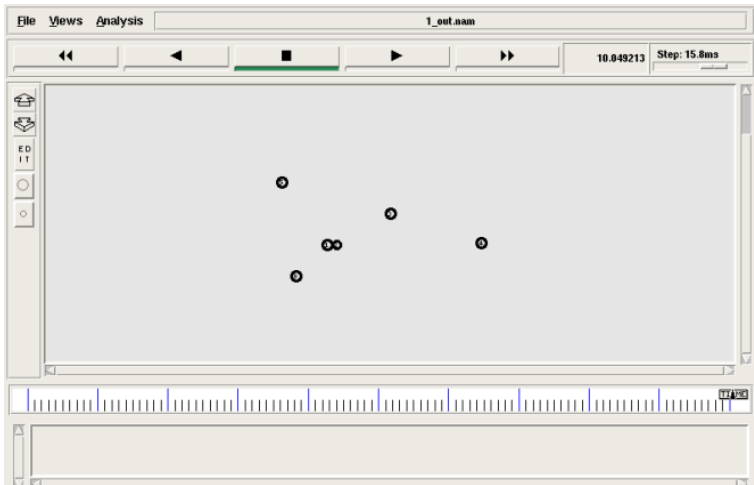

Fig. 9

This state is the state of extreme delay or this can be stated as state of confusion that may arise due to non decision of relaying node. In general, we can say the transmitter is unable to decide which node to pick for transmission purpose thus, in the actual time of transmission is lost and the network structure gets defaulted. Thus to prevent this situation, we develop some rules for transmission when mobility is of key concern. We termed this protocol as ECEP protocol which stands for AD HOC ON DEMAND DELAY ELIMINATION PROTOCOL. The protocol has the feature that a node is given a sensing facility by transmitting some signals at regular interval of time. We termed these signals as POSITION INDICATING SIGNALS. When a node receives such signals from a node which is making some transmission, the immediate location of that node is computed and if any of the node comes in the path, it at once changes it position toward another direction leaving the process of transmission unaffected. This state in our definition is termed as STATE OF TRANS. This is achieved only when a protocol has the capacity to judge the location on real time basis. This can be shown easily as follows:

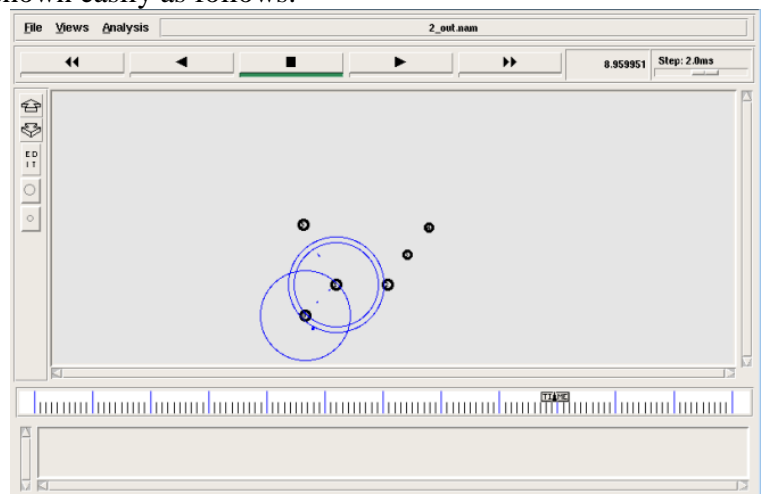

Fig. 10

When the node starts moving towards the relaying nodes from its already changed locations as compared to Fig. 9, it reaches a position where it catches the POSITION INDICATING SIGNAL from the relaying node and this is where the STATE OF TRANS occurs.

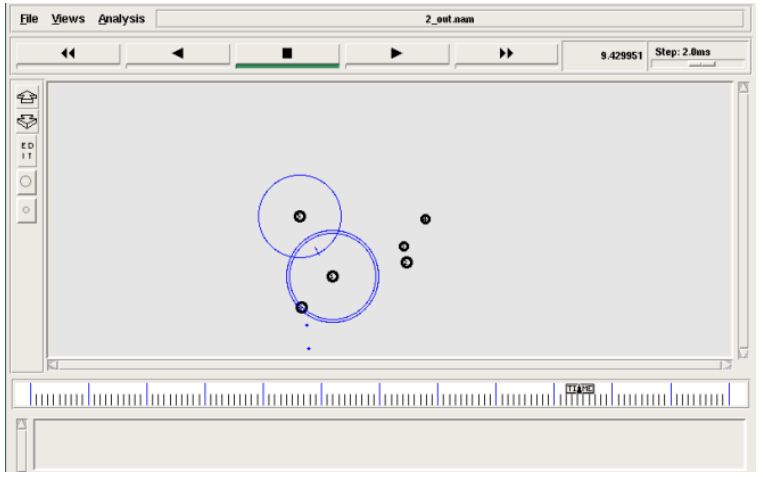

Fig. 11

The node that received POSITION INDICATING SIGNAL from the relaying node immediately changed its direction leaving the actual transmission process unaffected. This is one of the major features of the delay elimination protocol. The ECEP protocol developed by us also uses the technique of SNCP i.e. Sub Network Control Protection that causes ring formation to carry out transmission on zero delay basis. This protocol uses the delay elimination algorithm that we computed by running out the simulation in different scenario and finally coming out with the generalized algorithm theme as follows:

\subsection{Cluster Elimination Algorithm}

While (1)

\{

ring_search (i)

if $($ node $==$ true $)$

\{

then table_entry=valid

delay (node)

\} else

\{

Actual_path=true

Exit (0)

\}

\}

Delay (n)

\{

For $\mathrm{n}=0$ to node

\{

If (node_found==true)

\{

Apply cluster elimination code on head node

Select number of relay nodes

Maintain table and header entries

Exit (0)

\}

Else

\{

No_change

Exit (0)

\}

The above algorithm first traverses all the nodes present in the network structure. After the traversal of nodes, it is verified if most optimum path is selected or not. Then the delay tolerance is checked and on account of delay, the node is selected to act as host for SNCP and the relaying nodes are selected on basis of path that has its energy greater than the threshold energy, i.e. the energy actually required for purpose of transmission. 


\section{PERFORMANCE METRICS}

We simulated the typical MANETs for hop and calculated the results for delay variation, bandwidth, energy, throughput of the network structure taking mobility and the antenna range as the basis of the technique. We have taken values as constant to compute our results. The simulation has been performed for the area ranging over $1500 \times 1500$. Also, the values for the transmitter energy are taken to be $0.38 \mu \mathrm{J}$ and that of receiver processing energy is $0.25 \mu \mathrm{J}$. The simulation carried by us was taken on different scenarios taking different number of nodes at different geographical area to allow maximum zero error simulations. We concentrated on the following performance metrics:

- Delays: the delay is the latency caused in the process of transmission between the source and the destination. The delay considered in this paper is those that arise due to switching of the path to alternative route.

- Energy Efficiency: Energy efficiency is the efficiency computed over the total energy consumption of the network including the useful energy and the wasted in form of losses during the transmission process. This is calculated as the modified energy for the network structure.

- Bandwidth efficiency: Bandwidth efficiency is the effective rate of transmission that can be considered at the common rate as well as at the common power. The effective rate i.e. the bandwidth is computed over the minimum achievable rate and the number of channel used for the purpose of transmission between the source and the destination. The value considered for minimum achievable rate over which bandwidth efficiency is calculated is 0.25 .

- Throughput: throughput is the number of bits transferred during the transmission process. It is calculated I bits transferred per unit time. For our analysis the time considered is the simulation time.

- Mobility: Mobility is the new parameter that is highlighted in this paper. The readings have been taken by considering the scenario to be mobile and animations has also been recorded for the mobile nodes.

- Packet Size: The packet size is the number bits being processed during one set of transmission between the receiver and the transmitter. It is computed over end to end distance.

- Number of Re-Transmissions: The number of retransmissions is the packets that are to repeatedly transmit when failure occurs due to transmission delays.

\section{GRAPHICAL ANALYSIS AND RESULTS}

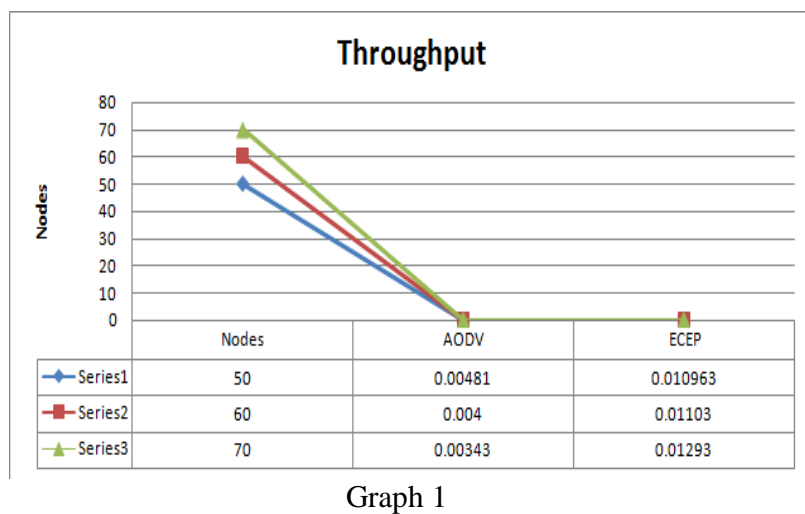

The graph 1 explains the throughput comparison between the ECEP and the AODV protoco measured in number of bits transerred per unit joule per unit time.

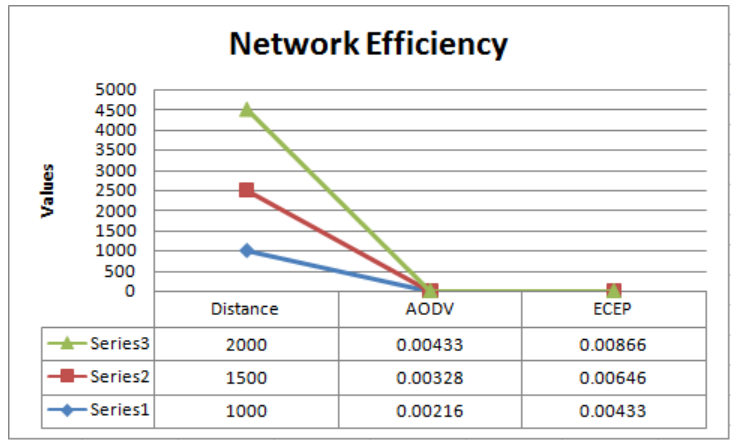

Graph 2

The graph 2 explains the graphical compariosn betweent he network effieicny of the AODV and the ECEP protocol.

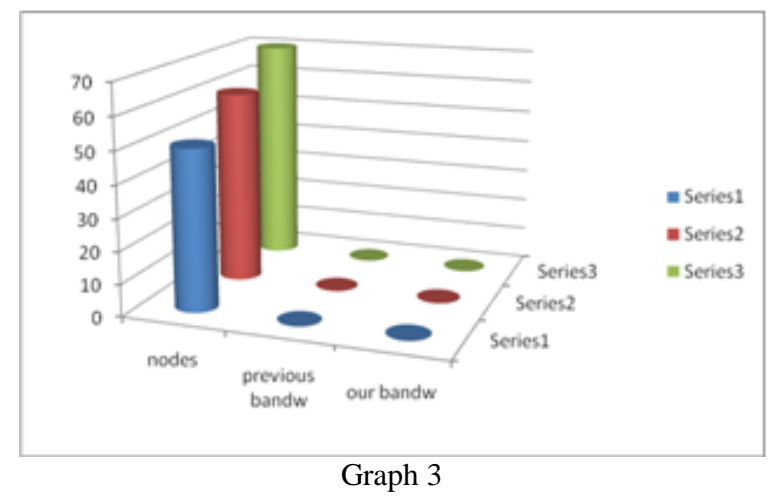

The Graph 3 explains the comparison between the AODV and ECEP's Network Bandwidth. The series 3 is the result of our protocol as compared to AODV in series 2 on basis of number of nodes in series 1 .

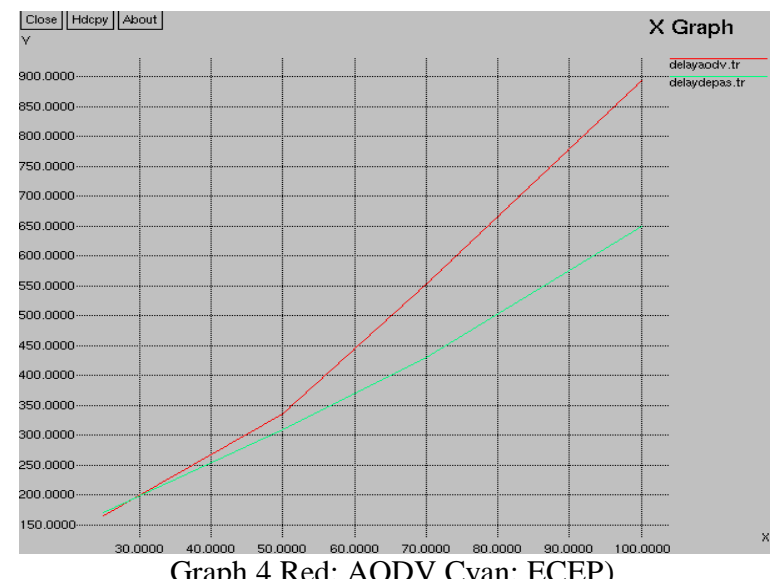

Graph 4 shows the relation between the actual delays in ECEP and the AODV protocol. Clearly, the ECEP stands out to be better in the comparison of the two. 


\section{CONCLUSIONS}

Thus, from the paper, it is noticed that in order to increase the performance of the network, the delays of the system must be increased. In this paper, we have proposed ECEP protocol. The protocol proposed in the paper is capable of handling the cluster formation that further drops the amount of delays thus improving the efficiency of the network structure. In future, the work can be compared by taking the practical values by surveying the network structure in the practical environment.

\section{REFERENCES}

[1] Dr. R.K. Singh, Tanu Preet Singh, Vishal Sharma, "Dead State Recovery Based Power Optimization Routing Protocol for MANETs," HPAGC-2011, CCIS 169, () Springer-Verlag Berlin Heidelberg-2011, pp.424-429.

[2] Sunil Taneja and Ashwani Kush, "A Survey of Routing Protocols in Mobile Ad Hoc abstract," International Journal of Innovation, Management and Technology, Vol. 1, No. 3, August 2010.

[3] M.Saravana karthikeyan, M.Murali, Dr.S.Sujatha,"Identifying performance metrics to maximize Manet's throughput,"2010 International Conference on Advances in Computer Engineering.

[4] Manika Vinay Rali, Min Song, Sachin Shetty, "Virtual wired transmission scheme using directional antennas to improve energy efficiency in Wireless Mobile Ad Hoc Network," 978-1-4244-2677-5, IEEE 2008.

[5] http://www. csi.uoregon.edu.

[6] Sehoon Kim, Jinkyu Lee and Ikjun Yeom, "Modeling and Performance Analysis of Address Allocation Schemes for Wireless sensor networks," IEEE transactions on vehicular technology, vol. 57, no. 1, January 2008.

[7] Rekha Patil, Dr. A. Damodaram, "Cost Based Power Aware Cross Layer Routing Protocol For Manet,"2008 IJCSNS.
[8] Changchun Bae and Wayne E. Stark, "A Tradeoff between Energy and Bandwidth Efficiency in Wireless Networks," 2007 IEEE.

[9] V. Rodoplu and T. H. Meng, "Bits-per-Joule capacity of energy-limited wireless networks," IEEE Transaction Wireless Communications, vol.6(3), March 2007, pp.857-865.

[10] B. Rankov and A. Wittneben,"Spectral efficient protocols for half-duplex fading relay channels," IEEE Journal on Selected Areas in Communications, vol. 25, Feb. 2007, pp.379-389.

[11] Network simulator-2 www.isi.edu/nanam/ns/.

[12] H. Wang and E.Stark, "transport Efficiency for Wireless Network",IEEE 2004.

[13] R. Zheng and R. Kravets:" On-Demand Power Management for Ad Hoc Networks". In Proc. IEEE INFOCOM, 2003, , pages 481-491.

[14] Wing Ho Yuen, Chi Wan Sung:”On energy efficiency and network connectivity of mobile ad hoc network". Proceedings of the 23rd International Conference on Distributed Computing Systems (ICDCS'03).

[15] W. E. Stark, H. Wang, A. Worthen, S. Lafortune, and D. Teneketzis: "Low-energy wireless communication network design," IEEE, Wireless Communications, Aug. 2002, pp.60-72.

[16] Wayne Stark, Hua Wang, Andrew Worthen, Stephane Lafortune, and Demosthenis Teneketzis:" low - energy wireless communication Network Design": IEEE 2002.

[17] P. Gupta and P. R. Kumar, "The capacity of wireless networks:" IEEE Transaction Information Theory, and vol. 46 (2), Mar 2000, , pp.388-404.

[18] S. Verdu: "On channel capacity per unit cost," IEEE Transaction Information Theory and vol. 36(5), Sept 1990, pp.1019-1030. 